\title{
Electronic, Magnetic, and Optical Properties of Double Perovskites $\mathrm{Pb}_{2} \mathrm{XOsO}_{6}$ (X=Co, Ni)
}

Bishnu P. Belbase ${ }^{1,2}$, Shalika R. Bhandari ${ }^{1,2,3}$, Madhav P. Ghimire ${ }^{1,2,3}$

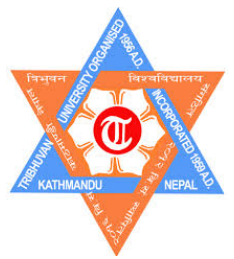

${ }^{1}$ Central Department of Physics, Tribhuvan University, Kirtipur 44613, Kathmandu, Nepal

${ }^{2}$ Condensed Matter Physics Research Center, Butwal, Rupandehi, Nepal

${ }^{3}$ Leibniz Institute for Solid State and Materials Research Dresden, Helmholtz str. 20, 01069

Dresden, Germany 


\section{Outline}

- Introduction

- Methods and Computational Details

- Crystal Structure

- Results and Discussion

- Conclusions 


\section{Introduction}

Materials Type

- Metals

- Half-metals

- Semiconductors

- Insulators

\section{Double Perovskites (DPs)}

- Most common DPs are $\mathrm{A}_{2} \mathrm{BB}^{\prime} \mathrm{X}_{6}$

- DPs covers all types of materials

- Shows remarkable properties like structural stability, superconductivity, half-metallicity etc
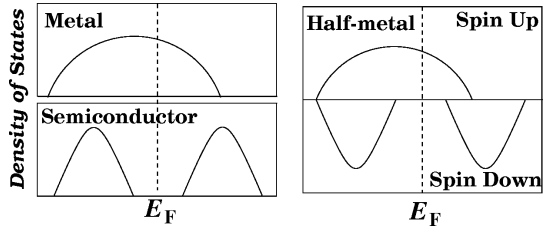

Fig: Classification of materials according to their energy gap [I. Galanakis et al., arXiv preprint cond-mat/0510276 (2005)]

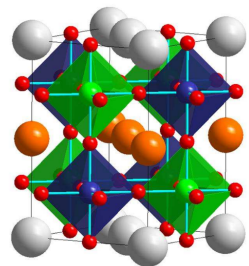

Fig: Ideal DPs structure [K. Momma et al., J. Appl. Crystallogr. 44, $1272(2011)]$ 


\section{Introduction}

Applications

- Spintronics devices

- Photovoltaic application

- Laser light and light-emitting diode

- Microelectronics and telecommunication

Osmium based DPs

- $\mathrm{Sr}_{2} \mathrm{CrOsO}_{6} \rightarrow$ Half metallic antiferromagnet ${ }^{1}$

- $\mathrm{Ba}_{2} \mathrm{NiOsO}_{6} \rightarrow$ Dirac-Mott insulating ferromagnet ${ }^{2}$

- $\mathrm{Ca}_{2} \mathrm{CrOsO}_{6} \rightarrow$ Ferrimagnetic insulator ${ }^{3}$

- $\mathrm{SrLaFeOsO}_{6} \rightarrow$ Metallic antiferromagnet ${ }^{4}$

${ }^{1}$ K. W. Lee et al., Phys. Rev. B 77, 115101 (2008)

${ }^{2}$ H. L. Feng et al., Phys. Rev. B 94, 235158 (2016)

${ }^{3}$ S. R. Bhandari et al., RSC Adv. 10, 16179 (2020)

${ }^{4}$ D. K. Yadav et al., Comput. Mater. Sci. 170, 109168 (2019) 


\section{Methods and Computational Details}

Theoretical approach

- Performed density functional calculation to explore electronic and magnetic properties

- Used GGA functional

- $\mathrm{GGA}+U$ functional considered to account strong correlation effect of Co-3d, $\mathrm{Ni}-3 d$ and Os-5d

- $U$-value for $\mathrm{Pb}_{2} \mathrm{CoOsO}_{6} \rightarrow \mathrm{Co}=4 \mathrm{eV}$ and $\mathrm{Os}=2 \mathrm{eV}$

- $U$-value for $\mathrm{Pb}_{2} \mathrm{NiOsO}_{6} \rightarrow \mathrm{Ni}=5 \mathrm{eV}$ and $\mathrm{Os}=2 \mathrm{eV}$

Computational details

- Full potential local orbital (FPLO) code is used for calculation

- $K$-mesh used for band structure and density of state $\rightarrow 12 \times 12 \times 10$

- $K$-mesh used for optical properties $\rightarrow 20 \times 20 \times 20$

- Convergence criteria: Energy $\rightarrow 10^{-8}$ Hartree and charge convergence $\rightarrow 10^{-6}$ electronic charge 


\section{Crystal Structure}

$\mathrm{Pb}_{2} \mathrm{CoOsO} \mathrm{O}_{6}$

Crystal Information:

- Space group- 14 (P121/c1

$\rightarrow$ monoclinic)

- Lattice parameters:

$a=5.6365 \AA ; b=5.5836 \AA$;

$c=7.82833 \AA$

- Angles:

$\beta=89.815^{\circ} ; \alpha=\gamma=90^{\circ}$

- By replacing $\mathrm{Co}$ by $\mathrm{Ni}$ from $\mathrm{Pb}_{2} \mathrm{CoOsO}_{6}, \mathrm{~Pb}_{2} \mathrm{NiOsO}_{6}$ is formed.

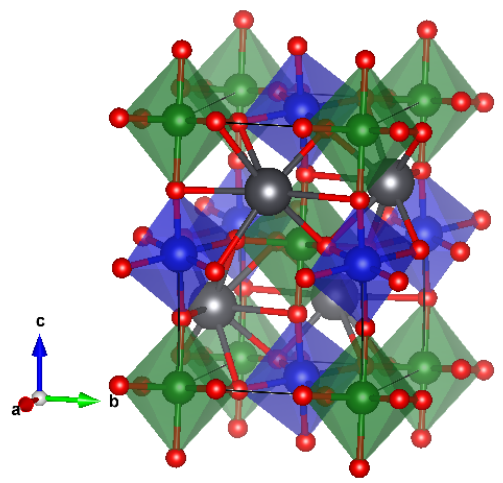

Fig: Crystal structure of $\mathrm{Pb}_{2} \mathrm{XOsO}_{6}(\mathrm{X}=\mathrm{Co}, \mathrm{Ni})$. Here green octahedra are due to $\mathrm{XO}_{6}$ and blue octahedra are due to $\mathrm{OsO}_{6}$. 


\section{Results and Discussion}

\section{Ground State and Magnetic Moment}

- Performed energy calculation for different magnetic configurations: one ferromagnetic state (FM- $\uparrow \uparrow \uparrow \uparrow)$ two antiferromagnetic states (AF1- $\downarrow \uparrow \uparrow \downarrow, A F 2-\uparrow \downarrow \downarrow \uparrow)$, two ferrimagnetic states (FI1- $\uparrow \downarrow \downarrow, F I 2-\uparrow \uparrow \uparrow \downarrow)$, and non-magnetic state.

- $\mathrm{Pb}_{2} \mathrm{CoOsO}_{6}$ has antiferromagnetic $(\mathrm{AF} 2-\uparrow \downarrow \downarrow \uparrow)$ ground state.

- $\mathrm{Pb}_{2} \mathrm{NiOsO}_{6}$ has ferrimagnetic $(\mathrm{Fl} 1-\uparrow \uparrow \downarrow \downarrow)$ ground state.

Table: Magnetic moment of $\mathrm{Pb}_{2} \mathrm{CoOsO}_{6}$
Table: Magnetic moment of $\mathrm{Pb}_{2} \mathrm{NiOsO}_{6}$

\begin{tabular}{|l|l|l||l|l|l|}
\hline Atoms & $\mathrm{GGA}\left(\mu_{B}\right)$ & $\mathrm{GGA}+U\left(\mu_{B}\right)$ & Atoms & $\mathrm{GGA}\left(\mu_{B}\right)$ & $\mathrm{GGA}+U\left(\mu_{B}\right)$ \\
\hline Co & 2.68 & 3.00 & $\mathrm{Ni}$ & 1.47 & 1.96 \\
\hline Os & 0.84 & 1.63 & Os & 1.32 & 1.69 \\
\hline Total & 0.00 & 0.00 & Total & 0.01 & 0.00 \\
\hline
\end{tabular}




\section{Results and Discussion}

Density of State

- $\mathrm{Pb}_{2} \mathrm{CoOsO}_{6}$ is metal and $\mathrm{Pb}_{2} \mathrm{NiOsO}_{6}$ is half metal.

- No contribution of Co and $\mathrm{Ni}$ around Fermi level.

- Strong hybridization between Os-5 $d$ and O-2pnear the Fermi level.

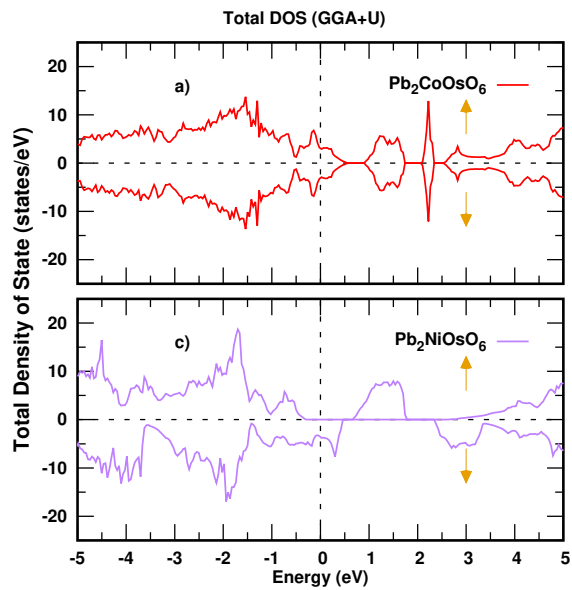

Fig: Total density of state of (a) $\mathrm{Pb}_{2} \mathrm{CoOsO}_{6}$ (b) $\mathrm{Pb}_{2} \mathrm{NiOsO}_{6}$ within $\mathrm{GGA}+U$. 


\section{Results and Discussion}

Partial density of State

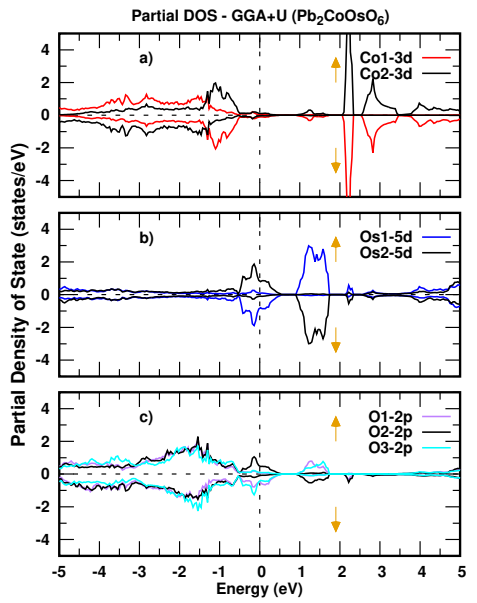

Fig: Partial density of state of $\mathrm{Pb}_{2} \mathrm{CoOsO}_{6}$ within $\mathrm{GGA}+U$.

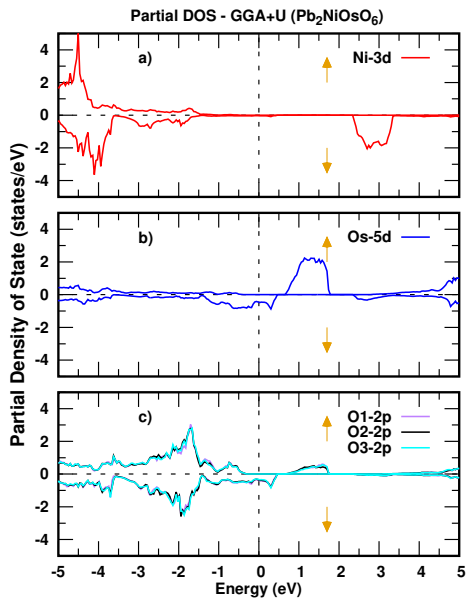

Fig: Partial density of state of $\mathrm{Pb}_{2} \mathrm{NiOsO}_{6}$ within $\mathrm{GGA}+U$. 


\section{Results and Discussion}

Band Structures

- Metallic nature in $\mathrm{Pb}_{2} \mathrm{CoOsO}_{6}$ and half-metallic nature in $\mathrm{Pb}_{2} \mathrm{NiOsO}_{6}$.

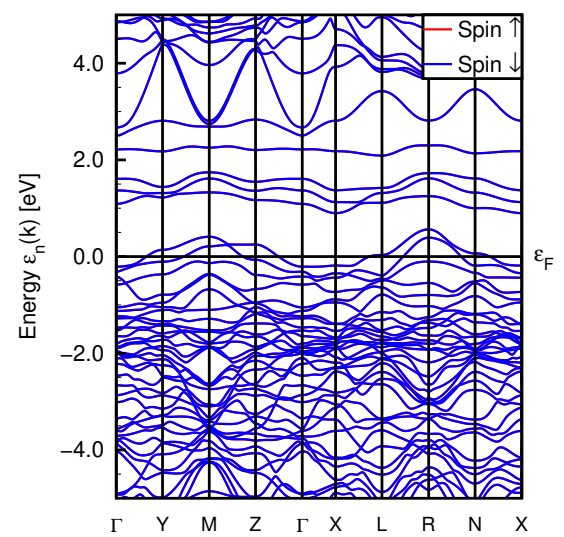

Fig: Band structures of $\mathrm{Pb}_{2} \mathrm{CoOsO}_{6}$ within $\mathrm{GGA}+U$.

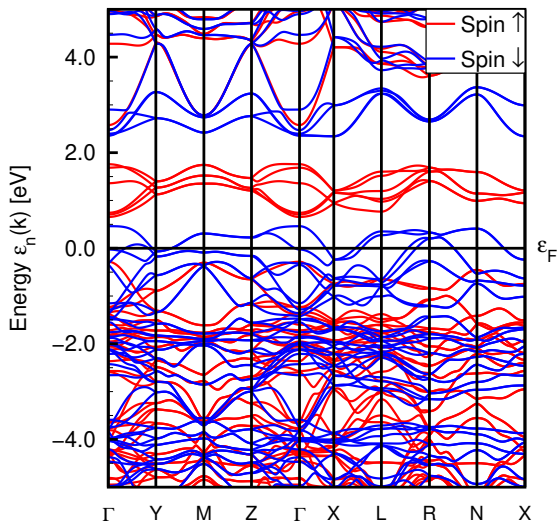

Fig: Band structures of $\mathrm{Pb}_{2} \mathrm{NiOsO}_{6}$ within $\mathrm{GGA}+U$. 


\section{Results and Discussion}

\section{$\mathrm{Pb}_{2} \mathrm{CoOsO}_{6}$}

- Optically active in the energy range $\rightarrow 0-6.4 \mathrm{eV}$

- Significant intraband transition
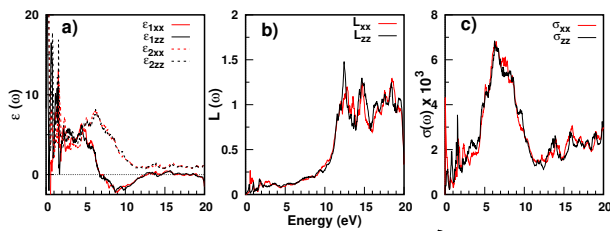

Fig: a) real $\left(\varepsilon_{1}\right)$ and imaginary $\left(\varepsilon_{2}\right)$ part of dielectric function, b) Loss function c) optical conductivity $\left(\mathrm{ohm}^{-1} \mathrm{~cm}^{-1}\right)$ of $\mathrm{Pb}_{2} \mathrm{CoOsO}_{6}$ within $\mathrm{GGA}+U$.

\section{$\mathrm{Pb}_{2} \mathrm{NiOsO}_{6}$}

- Optically active in the energy range $\rightarrow 1.4 \mathrm{eV}-6.8 \mathrm{eV}$.
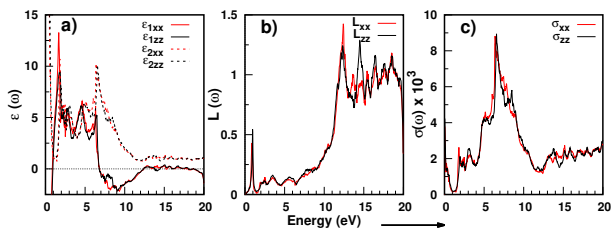

Fig: a) real $\left(\varepsilon_{1}\right)$ and imaginary $\left(\varepsilon_{2}\right)$ part of dielectric function, b) Loss function c) optical conductivity $\left(\mathrm{ohm}^{-1} \mathrm{~cm}^{-1}\right)$ of

$\mathrm{Pb}_{2} \mathrm{NiOsO}_{6}$ within $\mathrm{GGA}+U$. 


\section{Conclusions}

- $\mathrm{Pb}_{2} \mathrm{CoOsO}_{6}$ is antiferromagnetic metal whereas $\mathrm{Pb}_{2} \mathrm{NiOsO}_{6}$ is ferrimagnetic half-metal.

- With electron doping in $\mathrm{Pb}_{2} \mathrm{CoOsO}_{6}$, We found electronic and magnetic phase transition.

- We observed high intraband contribution in $\mathrm{Pb}_{2} \mathrm{CoOsO}_{6}$ as compare to $\mathrm{Pb}_{2} \mathrm{NiOsO}_{6}$

- $\mathrm{Pb}_{2} \mathrm{CoOsO}_{6}$ is optically active in infrared, visible, and lower energy range of the ultraviolet region.

- $\mathrm{Pb}_{2} \mathrm{NiOsO}_{6}$ is found to be more active in the visible region and lower energy range of the ultraviolet region. 


\section{Acknowledgments}

- Condensed Matter Physics Research Center, Butwal, Rupandehi, Nepal

- Sainamaina Municipality, Nepal

- Nepal Academy of Science and Technology (NAST), Nepal

- IFW Dresden, Germany
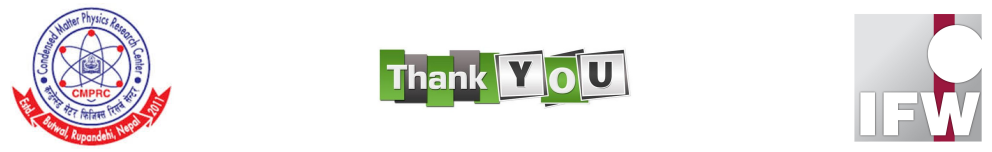